\title{
Evaluation of large area photomultipliers for use in a new Baksan Large Neutrino Telescope project
}

\section{Nikita Ushakov, ${ }^{*}$ Almaz Fazliakhmetov, Vladimir Gavrin, Tatyana Ibragimova, Bayarto Lubsandorzhiev, Arslan Lukanov, Alexander Shikhin, Andrei Sidorenkov and Dmitry Voronin}

Institute for Nuclear Research RAS,

pr. 60-letiya Oktyabrya 7a, Moscow, Russiy

E-mail: nikitaushakoff@gmail.com

The Baksan Large Neutrino Telescope is a liquid scintillation neutrino detector with a target mass of $10 \mathrm{kt}$, which is proposed to be constructed at the Baksan Neutrino Observatory of the Russian Academy of Sciences at a depth of 4700 m.w.e. In addition to the main full-scale detector, this project includes prototypes with target masses of 0.5, 5 and $100 \mathrm{t}$. This article evaluates 10-inch photomultipliers (PMTs) R7081-100 WA-S70 by Hamamatsu Photonics that are used in the first two phases of the project as the main ones. Also, this article evaluates 8-inch PMTs R5912-100 WA-S70, which are supposed to be used for the muon veto system of the 5-t detector prototype. To evaluate the PMTs, the quantum efficiency, single photoelectron response, timing characteristics, anode dark count rate, linearity and long afterpulses were measured.

$37^{\text {th }}$ International Cosmic Ray Conference (ICRC 2021)

July 12th-23rd, 2021

Online - Berlin, Germany

\footnotetext{
*Presenter
} 


\section{Introduction}

At the Baksan Neutrino Observatory of the Institute for Nuclear Research of the Russian Academy of Sciences (BNO INR RAS), it is planned to develop a large-volume neutrino detector based on a liquid scintillator with a volume of $10 \mathrm{kt}$. This multipurpose neutrino detector for detecting neutrino and antineutrino fluxes from the Sun, Earth and astrophysical sources will be located in the BNO underground zone at a depth of about 4700 m.w.e. (meter of water equivalent). The main goal of the project is to measure antineutrino fluxes from beta decays of isotopes of natural radioactive families ${ }^{238} \mathrm{U}$ and ${ }^{232} \mathrm{Th}$, as well as ${ }^{40} \mathrm{~K}$ contained in the interior of the earth (geoneutrinos). Reliable registration of these particles will make it possible to establish the contribution of the energy release from the radioactive decay of these isotopes to the total heat flux of the Earth [1]. Achieving this goal requires accurate measurement of the neutrino energy spectrum with extremely high energy resolution. In this regard, highly efficient photomultipliers (PMTs) are crucial for such large-scale neutrino experiments.

The detector project includes four stages: a prototype with a target mass of $0.5 \mathrm{t}$, a $5-\mathrm{t}$ prototype, a detector with a target mass of $100 \mathrm{t}$ and a full-scale detector for $10 \mathrm{kt}$. The first stage is completed [2]. At this stage, the detector uses twenty PMTs. The second stage, which is currently under construction, in addition to forty-two main PMTs, provides for the use of a muon veto.

The choice of PMTs for a detector based on a liquid scintillator was made among PMTs with bialkali photocathodes, which are most sensitive to near UV. The maximum possible coverage area of the detector with PMTs is required for the best energy resolution. Therefore, hemispherical PMTs were considered to improve the viewing angle. Modern PMTs are up to 20 inches in diameter, such as the R12860 by Hamamatsu and 20-inch microchannel plate PMTs (MCP-PMTs) by NNVT used in the JUNO experiment [3]. However, the dimensions of these PMTs are too large for prototypes of detector with target masses of 0.5 and 5 t. Modifications of 10-inch PMTs Hamamatsu R7081, R7081-100, were chosen as the main PMTs for these first two stages. The effective area of the photocathode of these PMTs is approximately 4.3 times smaller. However, an important advantage of these photomultipliers is their high quantum efficiency, which is about $35 \%$ at the peak at $390 \mathrm{~nm}$. Their quantum efficiency is $5 \%$ higher compared to R12860 (at a wavelength of $390 \mathrm{~nm}$ ). They also have a several times lower anode dark count rate, narrower pulses, and because of their smaller size, the effect of an external magnetic field on them is weaker. Single photoelectron (SPE) resolution, timing characteristics, quantum efficiency and dark count rate of 20-inch MCP-PMTs are slightly worse than R12860 [4].

In addition, the PMT R7081 has a different modification - R7081-20. This PMT has not 10, but 14 dynodes, and therefore has a greater gain (typical $10^{9}$ versus $10^{7}$ ). However, because of this, it has a higher anode dark count rate and worse timing characteristics, namely, more transit time spread and longer pulse duration and rise time. The decisive factor is that R7081-20 has a lower, by about $10 \%$ at $390 \mathrm{~nm}$, quantum efficiency.

Another PMTs with similar characteristics to the R7081-100 are the PMTs XP1802, XP1804, XP1805 and XP1807 by HZC Photonics. The most suitable of them, the 10.6-inch PMT XP1804, has the larger cathode area and the transit time spread of just 0.4 ns less than that of the R7081-100 (according to the data sheet). However, the quantum efficiency of all these PMTs is about $10 \%$ less than that of the R7081-100. 
Based on the mechanism of registration of muons and the design of the detector, photomultipliers for the muon veto are less demanding on the area of the photocathode. 8-inch PMTs Hamamatsu R5912-100 were chosen for the muon veto for the second stage of the project. Since the PMTs in the detector are located in water, for the R7081-100 and R5912-100, waterproof modifications with a 70-meter cable, R7081-100/R5912-100 WA-S70, were chosen. Further in this article, the R7081-100 and R5912-100 will mean exactly these modifications.

The quantum efficiency, single photoelectron (SPE) response, timing characteristics and anode dark count rate were measured for batches of twenty-five PMTs R7081-100 and twelve PMTs R5912-100. Linearity and afterpulses were also measured for the PMTs R7081-100.

\section{Quantum efficiency}

Quantum efficiency (QE) is one of the most important PMT parameters. Quantum efficiency is the number of photoelectrons emitted from the photocathode divided by the number of incident photons. The higher the quantum efficiency, the more photons can be detected and the better the energy resolution of the neutrino detector.

Measurement of the quantum efficiency consists in measuring the anode current when the PMT photocathode is illuminated with monochromatic light in a certain wavelength range. In this case, the PMT operates in a diode mode, that is, all dynodes, including the focusing ones, are shortcircuited to the anode. The following setup was used for measurements. Illumination in a wide wavelength range $(185-2000 \mathrm{~nm})$ from the powerful light source Hamamatsu E7536 with the xenon lamp L14286 was fed to the monochromator LM-4, which extracted light of a certain wavelength from white light; then this monochromatic light was fed to a PMT. The results are compared with a pre-calibrated photodetector. The Hamamatsu S1337-1010BQ silicon photodiode was used as a reference.

Quantum efficiency depends on the materials of the photocathode and dynodes, so it is approximately the same for all samples under consideration, including the R5912-100. Figure 1 shows the dependence of QE on the wavelength for the photocathode of the PMTs R7081-100 and R5912-100. According to the data obtained, QE exceeds $30 \%$ in the wavelength range from $330 \mathrm{~nm}$ to $450 \mathrm{~nm}$ and peaks at almost $40 \%$ at $390 \mathrm{~nm}$, which is excellent for the used scintillator with a maximum light output at $370 \mathrm{~nm}$.

\section{SPE response and timing characteristics}

The energy spectrum of a neutrino is measured by counting the total number of photoelectrons in each interaction of a neutrino with a scintillator. Therefore, the ability to measure a single photoelectron is a crucial requirement for PMTs of neutrino detectors. To achieve this, the PMT gain must be high enough. And also PMTs should be able to distinguish single photoelectron signals from noise, which requires that the peak of the SPE spectrum be narrow enough and have the largest possible peak-to-valley ratio. The setup for measuring SPE parameters was quite standard and included a digitizer and a LED, synchronized with the digitizer using a pulse generator. The 14-bit CAEN V1730 with a sampling rate of $500 \mathrm{MS} / \mathrm{s}$ was used as a digitizer. The LED had a wavelength of $405 \mathrm{~nm}$. 


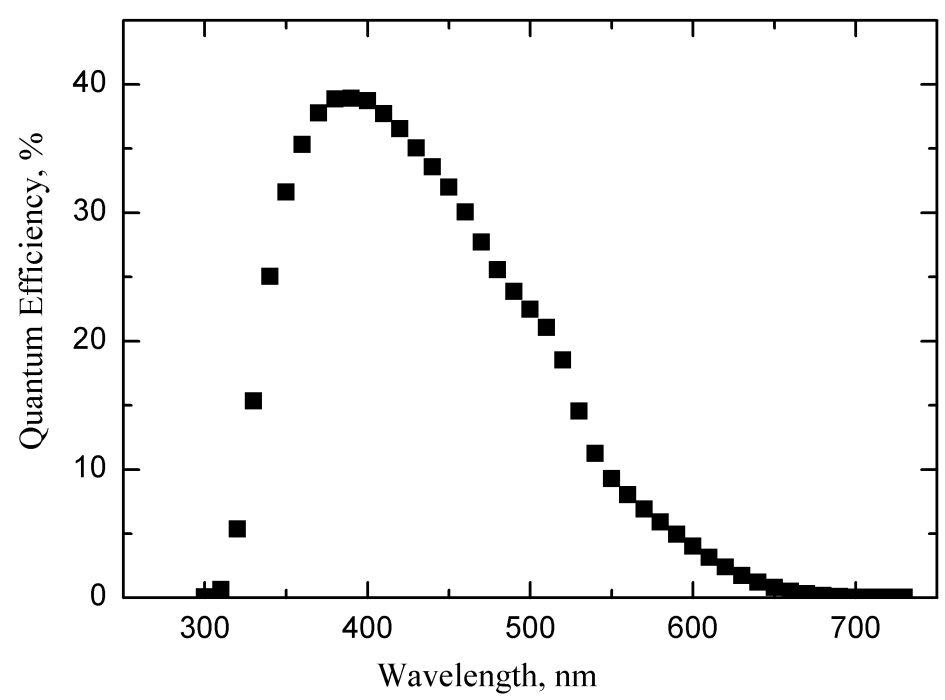

Figure 1: The dependence of QE on the wavelength for the PMTs R7081-100 and R5912-100.

Measurements were carried out with a PMTs gain of about $10^{7}$ and a fast amplifier gain of 10. In this case, the SPE pulse of the R7081-100 has the average amplitude of $45 \mathrm{mV}$ on $50 \mathrm{Om}$ terminating resistor; pulse duration is approximately $40 \mathrm{~ns}$ with rise time of $4.1 \mathrm{~ns}$. The SPE pulse of the R5912-100 has the average amplitude of $65 \mathrm{mV}$; pulse duration is approximately $25 \mathrm{~ns}$ with rise time of 3.9 ns. Figure 2 shows the final SPE signal waveforms of the PMTs R7081-100 and R5912-100.
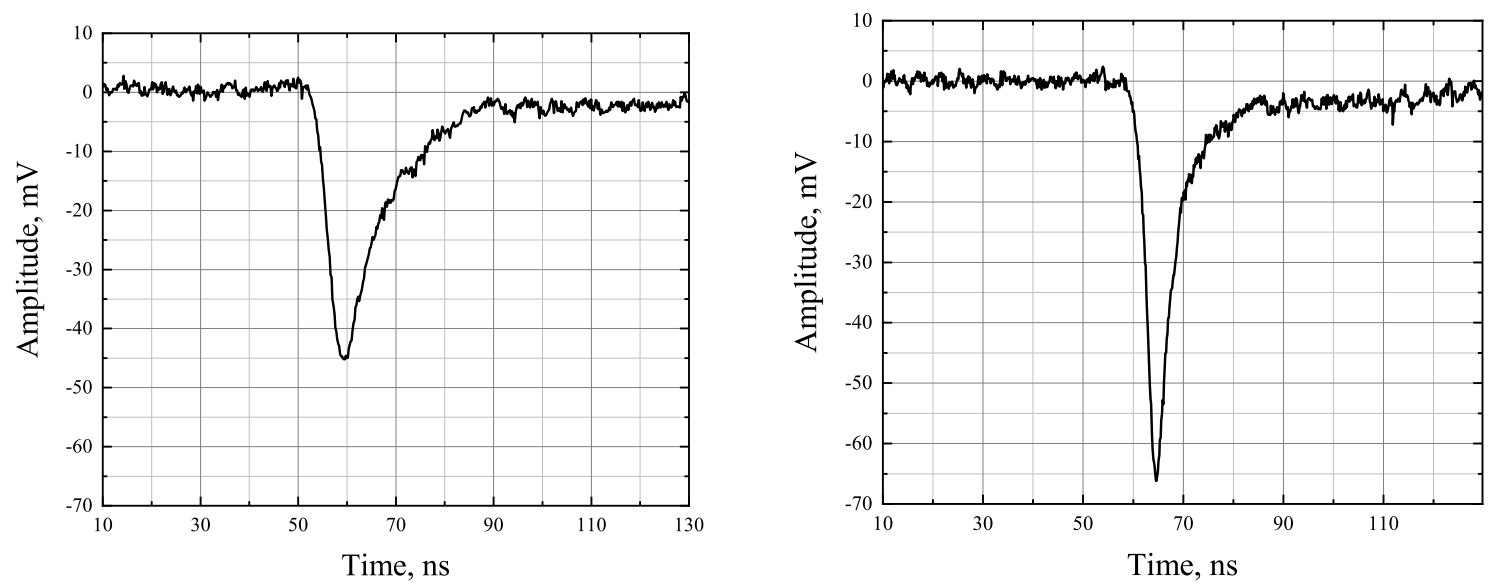

Figure 2: Single photoelectron pulse waveforms of the PMTs R7081-100 (left) and R5912-100 (right).

Figure 3 shows a typical SPE spectra of the R7081-100 and R5912-100. The average peakto-valley ratio of the R7081-100 is $3.73 \pm 0.56$ and SPE resolution is $0.68 \pm 0.06$. The average peak-to-valley ratio of the R5912-100 is $4.54 \pm 0.52$ and SPE resolution is $0.67 \pm 0.04$.

In addition, for such experiments, a transit time spread (TTS) is very important. The TTS is the fluctuation of the transit time of single photoelectron signals measured by PMT. Thus, the 

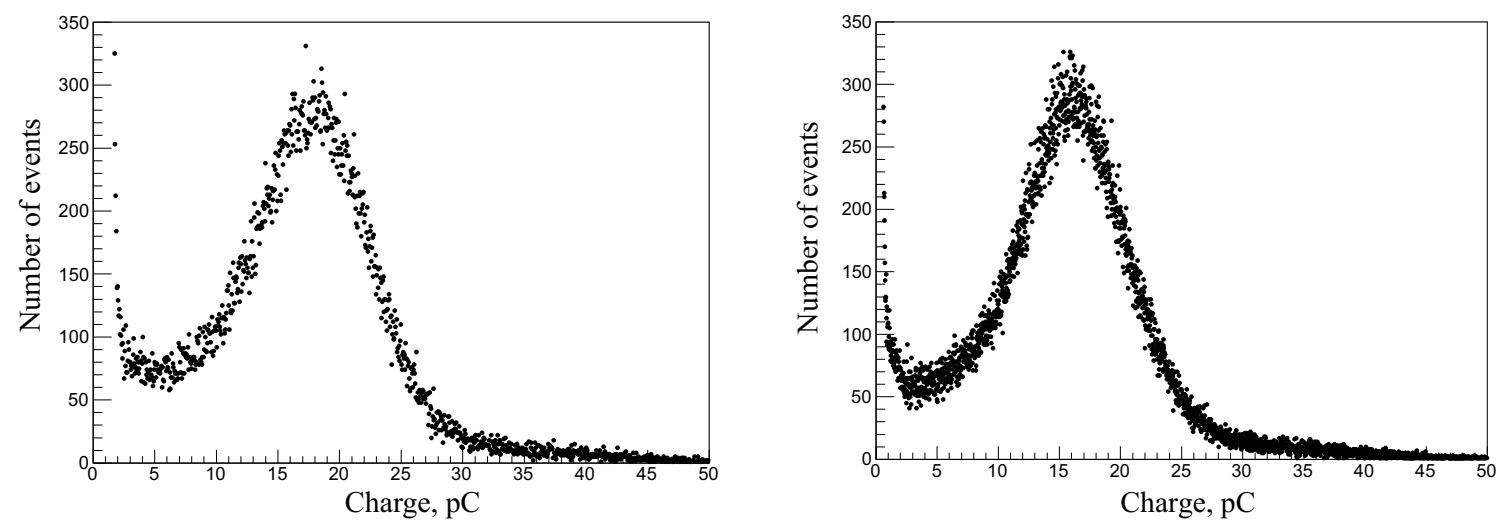

Figure 3: Typical single photoelectron spectra of the PMTs R7081-100 (left) and R5912-100 (right).

TTS imposes certain restrictions on particle tracking. Moreover, the photoelectrons can backscatter from the first dynode, are decelerated by the electric field and then accelerated again towards the first dynode, finally giving secondary electrons. These pulses are called delayed pulses [5]. Also, the photon can interact not with the photocathode, but directly with the first dynode; these pulses are called pre-pulses. The pre-pulses and delayed pulses can cause false triggers, complicate the processing of received events and can be confused with correlated events. The setup similar to that described above was used to measure the TTS of the PMTs. However, such measurements require a better time resolution, so the DRS4 with the sampling rate of $5 \mathrm{GS} / \mathrm{s}$ was used as a digitizer. The signal was supplied to the digitizer through a discriminator with a threshold of about $0.1 \mathrm{PE}$ for detect the pre-pulse. Figure 4 shows a typical TTS of the R7081-100 and R5912-100. The average jitter for the PMT R7081-100 batch is 3.01 $\pm 0.24 \mathrm{~ns}$ (FWHM); the pre-pulse is ahead of the main pulse by approximately $26 \mathrm{~ns}$, and the delayed pulse of elastic backscattered photoelectron lags by approximately $63.5 \mathrm{~ns}$. The average jitter for the PMT R5912-100 batch is $2.08 \pm 0.04 \mathrm{~ns}$; the pre-pulse is ahead of the main pulse by approximately $18 \mathrm{~ns}$, and the delayed pulse of elastic backscattered photoelectron lags by approximately $46 \mathrm{~ns}$.
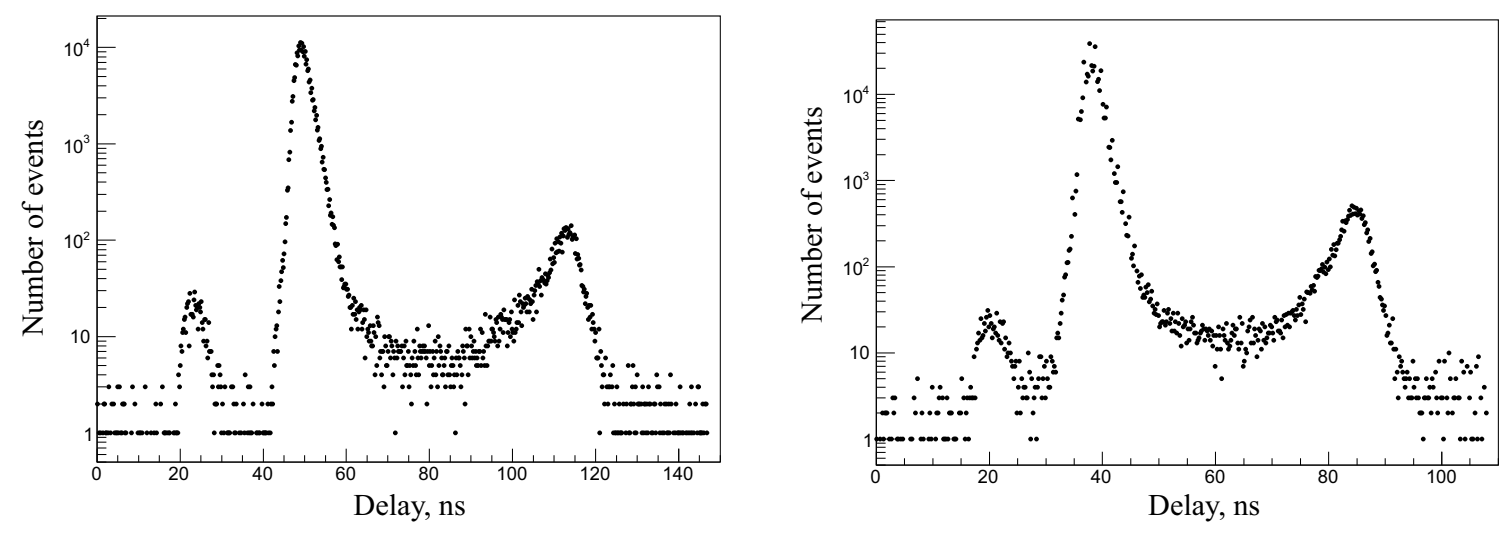

Figure 4: Typical TTS distributions of the PMTs R7081-100 (left) and R5912-100 (right). 


\section{Anode dark count rate}

The high noise level makes it difficult to identify events and correctly measure the energy of this event; despite the fact that the detector has a coincidence system. In a PMT, noise pulses can be defined as pulses that are not a photodetector response to a detected light event, that is, they occur even in complete darkness. The main source of such pulses is the spontaneous thermionic emission of electrons from the photocathode. The pulses resulting from this process generally correspond to a SPE. The dark rate is the rate of these noise pulses. The dark rate was measured with a frequency counter and discriminator with a trigger threshold of about 1/4 PE. The experiment was carried out at room temperature. The average dark rate value is $1.74 \pm 0.87 \mathrm{kHz}$ for the R7081-100 and $0.847 \pm 0.042 \mathrm{kHz}$ for the R5912-100, which is an excellent result for PMTs of this size.

\section{Linearity}

The intensity of the light flux from the scintillator can vary from several PE to several thousand PE, depending on the particle energy and the distance to the PMT. Therefore, the response of the PMT should be linear in a wide range. To measure the nonlinearity, two light sources similar to the aforementioned were used, whose signals were directed through the optical splitter to the PMT photocathode. The response of the PMT with simultaneous exposure of the photocathode by two LEDs was compared with the sum of the responses from each LED individually. A series of measurements was carried out, starting from low illumination intensities of the order of $10 \mathrm{PE}$ and ending with an intensity of about 900 PE. Figure 5 shows the curve of the expected and measured number of PE. Nonlinearity of 5\%, based on the data obtained for this type of photomultiplier, is achieved at a light intensity of approximately $330 \mathrm{PE}$.

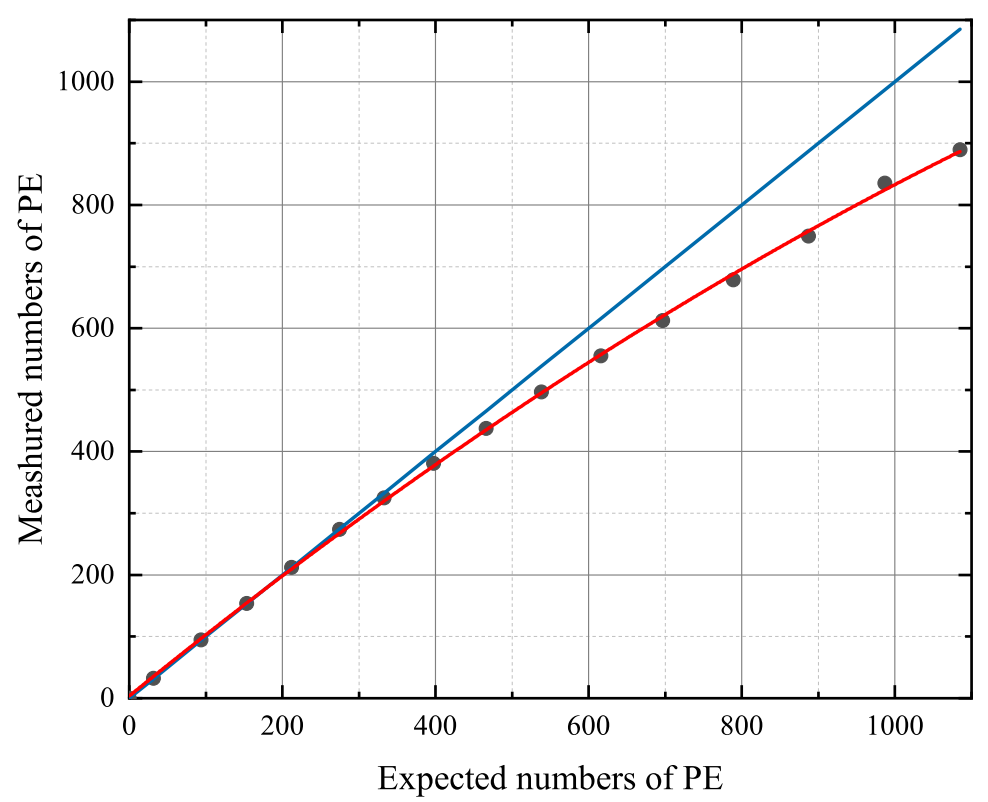

Figure 5: Typical curve of the expected and measured number of photoelectrons of the PMTs. 


\section{Afterpulses}

Afterpulses, like a pre-pulse and a delayed pulse, can cause false triggers and can be confused with correlated events. Long afterpulses are afterpulses caused by residual gas ions. The delay of such afterpulses from the main pulse can reach several microseconds. They were measured with illumination of the order of several tens of PE. Figure 6 shows a typical for the PMTs the distribution of long afterpulses delays taking into account the substrate from dark currents. After fitting (using the Landau distribution), contributions from ions of various gases to the final distribution were obtained. The ions are identified by calculating their transit time, $\Delta t$, using the electric potential distribution inside a hemispherical PMT. The calculated transit time assumes a quadratic potential such that,

$$
\delta t=\frac{\pi}{4} \sqrt{\frac{2 m}{q V_{0}}} L,
$$

where $V_{0}$ is the electric potential at the first dynode, $q$ and $m$ are the ion charge and mass, respectively, and $L$ is the distance to the photocathode. Table 1 shows the characteristics of each peak of this distribution; however, for each individual PMT, the indicated values may slightly vary depending on the concentration of a gas in the PMT and the operating voltage.

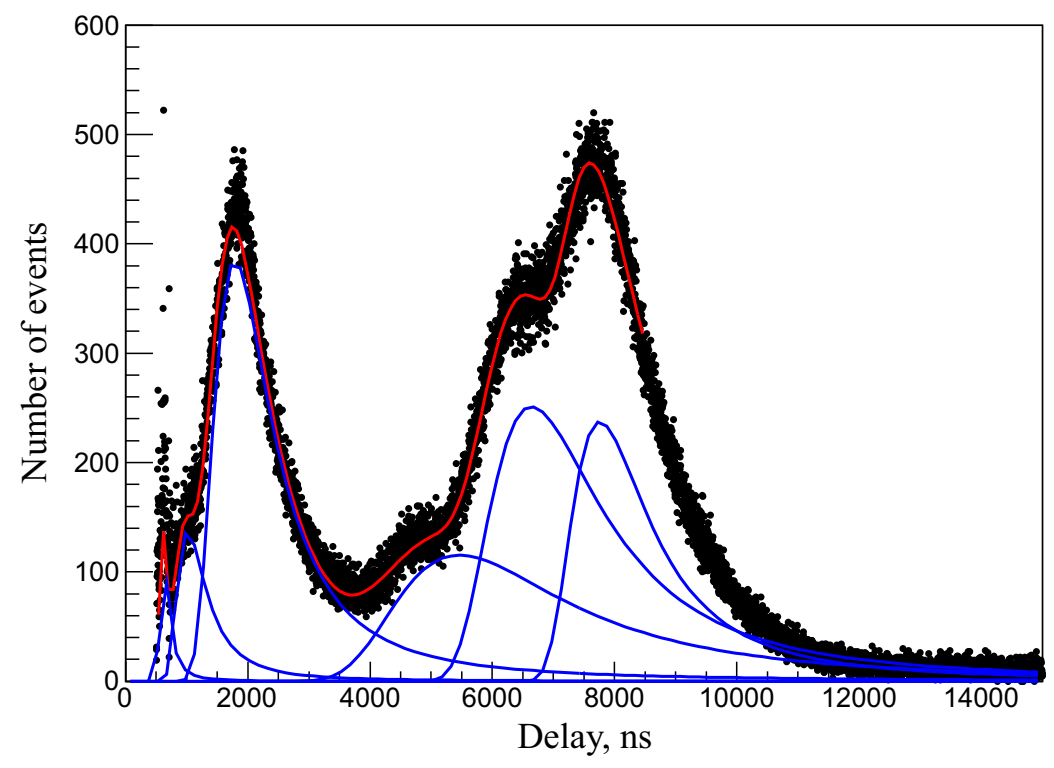

Figure 6: Typical delay distribution of long afterpulses for the PMTs and its fit.

\section{Summary}

Thus, this article briefly justifies the choice of 10-inch PMTs R7081-100 WA-S70 and 8inch PMTs R5912-100 WA-S70 by Hamamatsu Photonics for use in the Baksan Large Neutrino Telescope project. Also, this article evaluates batches of these PMTs of twenty-five and twelve pieces, respectively. The quantum efficiency exceeds $30 \%$ in the wavelength range from $330 \mathrm{~nm}$ to $450 \mathrm{~nm}$ and reaches a maximum of almost $40 \%$ at $390 \mathrm{~nm}$. The obtained values of peak-to-valley 
Table 1: Characteristics of the measured long afterpulses.

\begin{tabular}{lll}
\hline Peak position, $\mu \mathrm{s}$ & Possible source & The probability of occurrence, \% \\
\hline 0.605 & $\mathrm{H}^{+}$ & 0.02 \\
1.047 & $\mathrm{H}_{2}^{+}$ & 0.08 \\
1.854 & $\mathrm{He}^{+}$ & 0.44 \\
5.657 & Ions of some K-containing & 0.37 \\
& or organic compounds & \\
6.768 & $\mathrm{~K}_{2} \mathrm{O}^{+}$ & 0.53 \\
7.848 & $\mathrm{Xe}^{+}$ & 0.35 \\
\hline
\end{tabular}

ratio, SPE resolution, TTS and anode dark count rate are presented in table 2. The PMTs R7081-100 nonlinearity of $5 \%$ is achieved at a light intensity of about $330 \mathrm{PE}$. In addition, the distribution of the delays of long afterpulses was obtained; the probable sources and probability of occurrence for these afterpulses were identified.

Table 2: Characteristics of the measured PMTs.

\begin{tabular}{l|llll}
\hline & V/P ratio & SPE resolution & TTS (FWHM), ns & Dark rate, $\mathrm{kHz}$ \\
\hline R7081-100 & $3.73 \pm 0.56$ & $0.68 \pm 0.06$ & $3.01 \pm 0.24$ & $1.74 \pm 0.87$ \\
R5912-100 & $4.54 \pm 0.52$ & $0.67 \pm 0.04$ & $2.08 \pm 0.04$ & $0.847 \pm 0.042$ \\
\hline
\end{tabular}

\section{Acknowledgments}

The work was supported by the Russian Science Foundation, project No. 17-12-01331.

\section{References}

[1] L. Ludhova and S. Zavatarelli, Studying the Earth with Geoneutrinos, Adv. High Energy Phys. 2013 (2013) 425693.

[2] N.A. Ushakov et al., New large-volume detector at the Baksan Neutrino Observatory: Detector prototype, J. Phys. Conf. Ser. 1787 (2021) 012037.

[3] F. An et al., Neutrino physics with JUNO, J. Phys. G: Nucl. Part. Phys. 43 (2016) 030401.

[4] L. Ren et al., Mass production of MCP-PMT for JUNO and development of 20-inch MCP-PMT with TTS improved, JPS Conf. Proc. 27 (2019) 011014.

[5] B.K. Lubsandorzhiev, P.G. Pokhil, R.V. Vasiljev and A.G. Wright, Studies of prepulses and late pulses in the 8" electron tubes series of photomultipliers, Nucl. Instrum. Methods Phys. Res. A 442 (2000) 452. 